University of Nebraska - Lincoln

DigitalCommons@University of Nebraska - Lincoln

CSE Conference and Workshop Papers

Computer Science and Engineering, Department

2005

\title{
Ground Water Monitoring using Smart Sensors
}

Srikanth Anumalla

University of Nebraska-Lincoln

Byrav Ramamurthy

University of Nebraska-Lincoln, bramamurthy2@unl.edu

David Gosselin

University of Nebraska-Lincoln, dgosselin2@unl.edu

Follow this and additional works at: https://digitalcommons.unl.edu/cseconfwork

Part of the Computer Sciences Commons

Anumalla, Srikanth; Ramamurthy, Byrav; and Gosselin, David, "Ground Water Monitoring using Smart Sensors" (2005). CSE Conference and Workshop Papers. 69.

https://digitalcommons.unl.edu/cseconfwork/69

This Article is brought to you for free and open access by the Computer Science and Engineering, Department of at DigitalCommons@University of Nebraska - Lincoln. It has been accepted for inclusion in CSE Conference and Workshop Papers by an authorized administrator of DigitalCommons@University of Nebraska - Lincoln. 


\section{Ground Water Monitoring using Smart Sensors}

\author{
Srikanth Anumalla \\ and Byrav Ramamurthy \\ Department of Computer Science \\ and Engineering \\ University of Nebraska Lincoln \\ Lincoln, Nebraska 68588-0115 \\ Email: \{anumalla,byrav\}@cse.unl.edu
}

\author{
David C. Gosselin \\ and Mark Burbach \\ School of Natural Resources \\ University of Nebraska Lincoln \\ Lincoln, Nebraska 68588-0517 \\ Email: \{dgosselin2,mburbach\}@unl.edu
}

\begin{abstract}
Groundwater resources are the principal source of drinking water for about 50 percent of the United States population. Groundwater resources in Nebraska are under increasing stress as there is a rapid growth in their usage. Water managers need more timely and accurate data to assess ground-water conditions to manage adverse situations such as drought and loss of pumpage in agriculture and domestic water supply. Currently groundwater researchers use stand-alone data logging equipment which is labor intensive and the method is not easily scalable if the number of logging locations increases. Commercially available real-time data monitoring solutions are either prohibitively costly or highly inflexible.

In this paper we present a design for a groundwater monitoring system based on a network of wirelesslylinked pressure sensors. The proposed solution will enable groundwater researchers and decision makers to have quick access to the groundwater data with less effort and cost. Though our design is initially meant for groundwater monitoring, it can be easily adapted to other fields of environmental monitoring.
\end{abstract}

\section{INTRODUCTION}

Groundwater resources are the principal source of drinking water for about 50 percent of the United States population, providing nearly 96 percent of water used in rural domestic supplies and 40 percent in public supplies [2]. Groundwater resources in Nebraska are under increasing stress as there is a rapid growth in their usage. Water managers need more timely and accurate data to assess ground-water conditions to manage adverse situations such as drought and loss of pumpage in agriculture and domestic water supply. For example, drought severity and duration indices lack precision in detecting the onset, end, and accumulated stress of drought because they lack near real-time groundwater level data. At most locations, groundwater levels are commonly collected once or twice a year using a hand-held tape to measure the depth. At several hundred locations groundwater data are obtained by pressure transducers and recorded on an hourly to daily basis. Although these data are valuable to understanding the resource usage, they are not available in a timely fashion or at the frequency (days to weeks) that water resource managers need them to make relevant decisions. Limited real-time data can be obtained from remote locations using commercial solutions, but the cost of $\$ 3,500$ to $\$ 5,000$ per sampling location makes them prohibitively expensive to deploy at a state-wide or natural resources district level. In addition, the available commercial solutions lack the flexibility needed for our research infrastructure.

The need for real-time groundwater level data suggests the development of an infrastructure for acquiring, transferring and analyzing the real-time data. The proposed system should meet the following requirements.

1) Low cost: There are a huge number of locations where the groundwater level is measured. To deploy the infrastructure on a large scale requires that the cost of components and their integration should be less.

2) Miniaturization: Often the deployment environment for the sensors requires the sensors to be compact. The sensing intelligence must be implemented on high end integrated circuits to make them compact.

3) Flexibility: The design of the sensors should be flexible enough to be adaptable to other environmental measurements.

4) Battery Lifetime: As the cost and battery lifetimes are constrained, the design should be intelligent enough to manage the energy usage efficiently.

5) Modular Design: To enable accommodation for new technologies in various components (viz. data acquisition, transfer, and analysis) the design should be 
modular.

6) Reliable untethered communication: As the nodes are part of a network, the data transfer must be reliable in the event of node failures.

Currently available solutions for real-time data monitoring are accurate and reliable but they are inflexible and cost prohibitive. Line of sight is another problem for current solutions. Sensing devices and the base stations must be in the line of sight to enable communication which is not the case in most of the fields where the groundwater data is measured.

The advancement in sensor technologies, integrated circuits and data communications enable one to build a low cost yet reliable infrastructure for real-time monitoring of groundwater level data.

The outline of the rest of the paper is as follows. In Section II, we present relevant background and a discussion on earlier work. Section III addresses the design of the proposed solution followed by a discussion of the challenges involved in real-time groundwater monitoring. We discuss the status of our current work in realizing the proposed solution in Section IV, and finally we conclude the paper in Section V.

\section{BACKGROUND \& RELATED WORK}

In this section we present some of the issues in groundwater level monitoring, a brief overview of technologies used in our design such as field programmable gate arrays (FPGAs) and 802.11 Wireless LANs followed by a discussion on related earlier work.

\section{A. Groundwater Monitoring}

In order to critically assess the impact of drought on water resources in any region at any scale, one must have relatively quick access to the data.The data from this monitoring network can be studied with sophisticated information processing systems which refines the data at multiple temporal windows and spatial scales to derive support decisions. The groundwater level data will also be used to develop new drought indices. Severe drought is a recurring problem for the United States and has raised serious concerns about our nation's vulnerability to drought-induced water shortages. Drought results in serious economic, social and environmental impacts. The magnitude of future droughts may increase due to continued irrigation development, urban expansion, and expanding public water supplies. Global water consumption increased six-fold between 1990 and 1995, a rate twice that of population growth [13]. Two thirds of the world's population may experience water shortage by the year 2025 [13]. Thus, it is critical to develop an improved monitoring system to study water resource availability and usage.

Drought mitigation and monitoring programs have historically been reactive emphasizing emergency response [6], [4]. There have been serious limitations to efficient drought monitoring and response programs. Limitations include a lack of timely information provided to decision makers and other users on drought severity, lack of reliable and timely impact assessments, and lack of objective and timely designation procedures [5], [4]. Recent approaches to better combat drought emphasize preparedness, mitigation, and improved early warning systems [4]. New technologies, expanded monitoring networks and interagency collaborations offer opportunities for proactive drought preparedness programs and better informed management decisions.

Drought severity and duration indices are imprecise in detecting the onset, end, and accumulated stress of drought because they lack near real-time data [3]. Most drought indices also fail to differentiate the effects of drought on surface and subsurface water supply [3]. Traditional drought indicators such as the Standardized Precipitation Index (SPI) [7], [8] and the Palmer Drought Severity Index [9] have been useful in estimating the intensity and duration of meteorological drought; however, they are not truly designed to gauge the magnitude of droughts. Hydrological drought indicators have used point observations of water resources but have not been extended to spatial-temporal drought regions.

Large geographical areas, complex environmental systems and diverse anthropomorphic influences complicate the determination of the severity and duration of drought. It is critical for drought-prone regions to establish comprehensive and integrated drought early-warning systems that incorporate climate, soil and water supply factors such as precipitation, temperature, soil moisture, snowpack, reservoir and lake levels, streamflow and groundwater levels [4]. Of these components, in Nebraska, realtime or near real-time data is unavailable for lakes and groundwater levels.

Water-level measurements from observation wells are the principal source of information about the effects of hydrologic stresses on groundwater systems [1]. During times of severe drought this hydrologic stress is most acute. Watershed response to drought is variable - depending on such factors as precipitation, evapotranspiration, soil/geologic properties, runoff, and withdrawals. Surficial aquifers are highly vulnerable to droughtinduced hydrologic stress. While high production wells 
penetrating deep aquifers can significantly draw down water levels in those aquifers during drought, shallow groundwaters are more acutely responsive to drought and drought recovery. Drought or high precipitation can have an immediate and large impact on surficial groundwater [10], [11], [12].

\section{B. Commercial solutions}

We have considered one of the commercially available solutions for remote monitoring before we started to design a custom solution. Solinst [18] has "Solinst Telemtry System (STS)". STS enables 2-way communication and control between a base station and remotely located groundwater level loggers. The communication media can utilize cellular, GSM, CDMA, AMPS, land line telephone or radio. But this solution is prohibitively costly to deploy in large number of locations. One should use the software provided by the company to log the data and control loggers. To satisfy custom ways to control the data logging and logger operations one needs to have flexible interfaces to the system.

\section{Field Programmable Gate Array}

A Field Programmable Gate Array (FPGA) is a very complex Programmable Logic Device (PLD). The FPGA (for example, see Fig. 1) usually has an architecture that comprises a large number of simple logic blocks, a number of input/output pads, and a method to make random connections between the elements. The largest and fastest programmable logic devices have gate counts running into the millions. These devices are user customizable and programmable on an individual device basis. These are highly valued for their flexibility by designers [14].

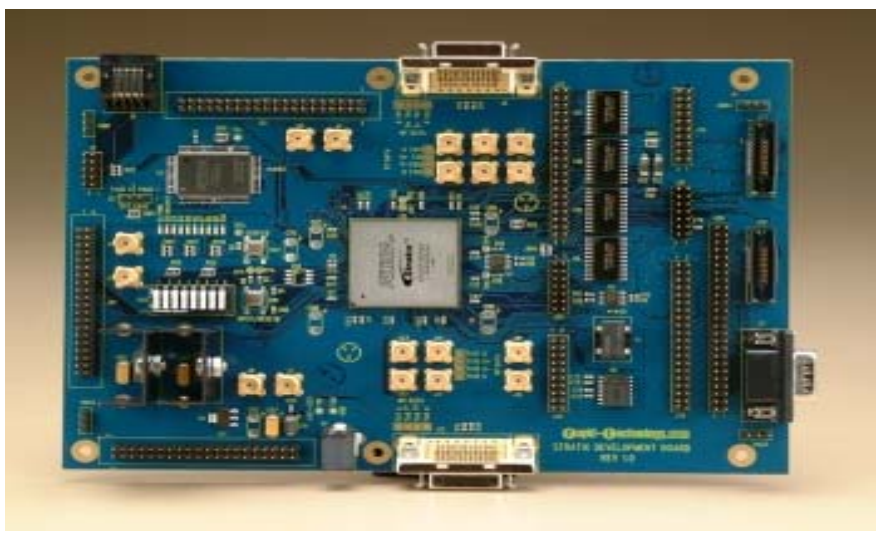

Fig. 1. Altera Stratix FPGA.

\section{Wireless LANs (802.11)}

The wireless local area network (WLAN) technology, IEEE 802.11 , typically provides a range of 50 meters with low-gain omnidirectional antennas. With high-gain antennas and line of sight we can extend the range upto 80-120 kilometers [15]. Though 802.11 networks have been used for setting up a home network or corporate network, they can be easily applied to environmental monitoring. With 802.11 networks, each node will have its own IP address and can be accessed or controlled over the Internet from a remote location.

\section{E. Related work}

The availability of compact sensor technologies has resulted in a profusion of applications in different domains. The use of sensors combined with wireless technologies has resulted in several devices for building wireless sensor networks.

1) Berkeley Motes: There has been lot of work carried out in wireless sensor networks. But most of the earlier work concentrated on short range wireless communication. One such example is Berkeley motes. At University of California-Berkeley, researchers have successfully integrated computation and communication in a cubic millimeter space. Smart Dust, as it is called, is self contained, millimeter scale sensing and communication platform for a massively distributed sensor network. These devices are inexpensive enough to be deployed on a large scale. These motes contain sensors, computational ability, bi-directional wireless communication, and a power supply. The limited range communication for these devices makes it inappropriate for measuring groundwater levels, where the different locations are separated by miles [17].

2) Reconfigurable Sensor Networks: One of the most relevant work for near real-time environment monitoring has been carried out by Brent et al. [16]. They have designed an integrated circuit, system and network to enable micro-climate monitoring. They have employed a multi hop routing scheme to enable data transfer using a Bellman-Ford like routing algorithm. This solution might suit applications where there is a low mobility of sensor devices. In the case of groundwater monitoring, sensors are static. We may not need a dynamic routing algorithm.

\section{Networking Smart Sensors}

The proposed infrastructure leverages the advancements in sensing technologies, integrated circuits and wireless communication infrastructure. There are three important aspects in our design - data acquisition, data 
transfer and data processing. Our priority is to minimize the total cost of the system while at the same time providing the amount of accuracy and reliability provided by the proprietary solutions. The system will also be generic enough to be adapted to other areas of environmental measurements. Our system synthesizes field programmable gate arrays and sophisticated 802.11 wireless networking infrastructure adapted to environmental sensing.

\section{A. Overview}

We divide the system into three important components each handing a specific task. Modularity accounts for the possibility of incorporating new advancements in each component without disturbing the whole system. Each component will have well defined interfaces. The components are the Data Acquisition Unit (DAU), the Data Transfer Unit (DTU) and, the Data Processing Unit (DPU). The DAU is responsible for collecting the data from the sensor and responds to data requests from the DTU. DTU will transport the data from the DAU to the DPU reliably. Finally, the DPU, after gathering the data from sensors, will analyze and store the data for end use. The following picture (see Fig. 2) depicts the total system.

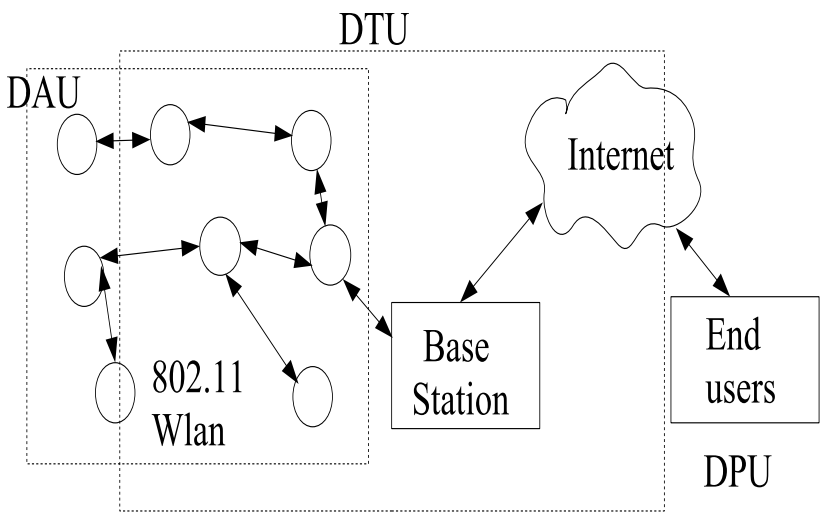

Fig. 2. Remote monitoring using 802.11.

A key idea of our project is to use off-the-shelf hardware and technology to build a low-cost, flexible data acquisition and transmission system that will be vendor-independent. We are developing two laboratorybased prototype systems, one that will interface with cell phone technology and the other that will interface with high-gain 802.11 wireless protocol. In the future, we will field-test the prototypes and assess their ability to provide data from a variety of locations and a variety of conditions.
We describe the three components of the system, in detail, below.

\section{B. Data Acquisition Unit}

The DAU (see Fig. 3) will consist of a sensor (a pressure transducer in this case) that is connected to a micro controller (FPGA) through an Analog to Digital Converter (ADC). The sensor will measure the pressure, which is related to the amount of water above it, and sends the analog signal to ADC. ADC will convert the analog signal to digital value and sends the data to micro controller using a pre-defined protocol, which we have developed. During the course of further improvement, we will need to consider the extent to which we will include the computation of temperature compensation in the DAU. The power for the devices can be provided either using solar panels or long-life batteries.

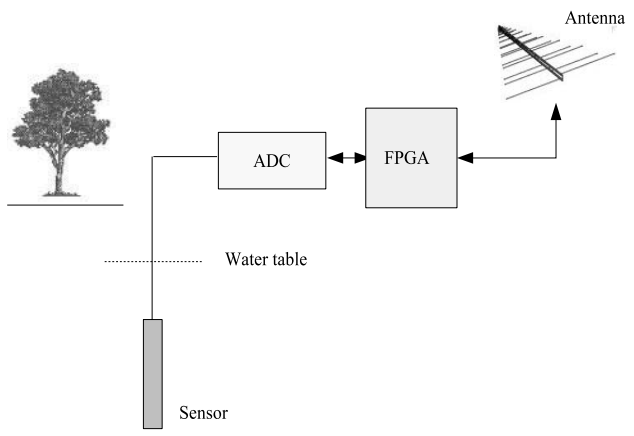

Fig. 3. Data Acquisition Unit.

\section{Data Transfer Unit}

The data transfer unit is responsible for carrying the data from the DAU to the DPU. Data transfer will be accomplished using cell phone technology and 802.11 wireless network protocols. Cellular technology enables the connectivity between remote location and the Internet. Cellular phone service currently has a limitation of availability and stability in remote locations across Nebraska. A high gain 802.11 network is potentially a better choice because it costs less and the user has more control. The 802.11 network will be based on TCP/IP network protocol thereby giving the ability to control the DAU to a remote operator. The use of intelligent routing protocols will enable sensors to form networks and thereby routing the information in the absence of direct line of sight to the base station. Our goal for both technologies is to transfer data in real time (once per day is considered to be real time) to the Data Processing Unit 
(DPU). The challenges that have to be addressed during DTU development are the stability of connectivity and data security.

\section{Data Processing Unit}

The data processing unit is where the data can be manipulated by analysts and/or made available to the public. New archiving and analysis techniques will be developed to handle the accumulation of potentially large amount of data being accumulated every day. Most components of DPU will consist of software that will allow the user to access the data graphically (see Fig. 4) or in a tabular format. We will also be considering future development of maps for data display. A key characteristic of any software that will be developed is that it be flexible and user friendly. The development of the DPU is currently ongoing.

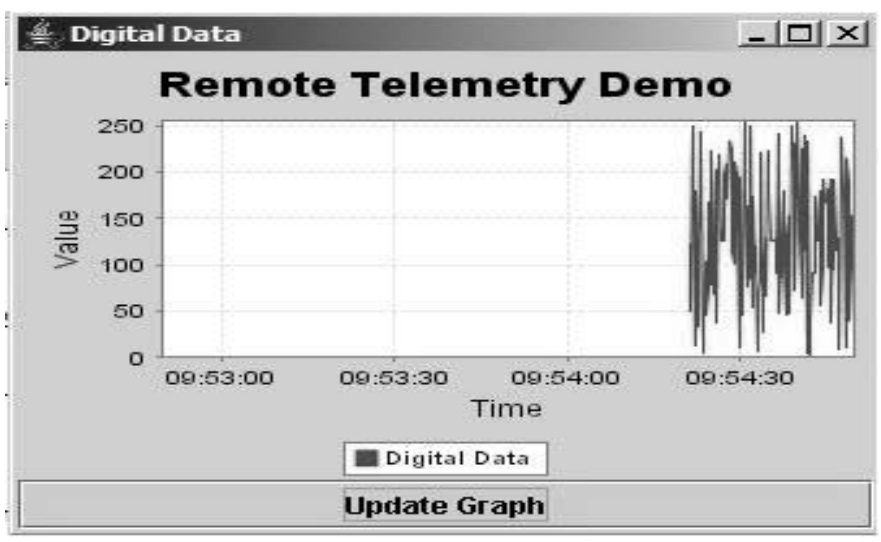

Fig. 4. Graphical display of data.

For the research to be complete, correct and effective, collaboration and cooperation among the experts in computer technology and water resource management is required. The design of such a system needs to be flexible enough so that it can be scaled to a variety of applications and we obtain stable connectivity.

The ultimate goal of this design is to deploy a statewide sensor network for groundwater monitoring using the more economically feasible system that we will develop. As the system is developed, our intent is that the DAU will be a generic programmable board that will interface with a wide range of sensors or data sources. The proposed research will provide the experience necessary to extend this work to other environmental monitoring applications. Our long-term vision is that this system will be able to be deployed in remote areas and provide a stable monitoring infrastructure that overcomes the physical limitations inherent in these environments.

\section{Current Work}

Currently a prototype implementation for the framework has been built that can be demonstrated in a lab environment. The components used for prototype are Unidata pressure sensor [19], Microchip's PIC12F675 micro controller [20], Altera Nios FPGA development board [21], Dlink's Ethernet-wireless bridge [22] and a Wireless base station. The DAU was implemented using a microchip microcontroller (PIC12F675) along with a pressure sensor. The groundwater level is measured using the pressure acting on the sensor located at some depth inside the water. The pressure sensor is connected to the analog port of the micro controller. The PIC12F675 microcontroller has an in-built $\mathrm{A} / \mathrm{D}$ converter. This micro controller is programmed to read its analog port periodically and to convert the analog signal to a 8 bit digital value. The digital data from the micro controller is communicated to Altera Nios FPGA processor through RS232 serial communication. The FPGA is programmed to perform two tasks. First, communicating with the microcontroller for obtaining the digital data of the measured pressure and communicating with a remote computer to transfer the data when requested. The DTU has been implemented as a long-range 802.11 ad-hoc network, along with Internet, enabling the connectivity between a computer and the DPU. As part of the DPU we have developed a software in Java which can request the data from a particular sensor and stores it in a database according to user preferences. The software also has a sophisticated interface to display the chosen data in graphical format. Future enhancements to our prototype will be tested in a real-world environment at Gudmundsen Ranch in Western Nebraska where large number of sensors have currently been deployed.

One of the key objectives of our groundwater monitoring project is to reduce the cost of the system. The prototype implementation costs around $\$ 400-\$ 500$ but the cost can be drastically reduced if all the components of the DAU are integrated on to one single electronic circuit. The integrated circuit (IC) can be deployed in challenging locations (such as inside wells) as it can be be made very compact. Our design leverages the TCP/IP protocol, which is the same protocol used for the Internet. With the generality provided by TCP/IP all the devices can be accessed from anywhere on the Internet. Moreover one can implement a web server on the DAU to provide a web interface to the devices, in which case the device can be accessed and controlled from a web browser. One important concern is the battery life of the 
power source enabling the devices. Right now our design assumes the use of solar panels. The battery life can be improved by designing the devices so that they can be turned off during non-operation times.

\section{CONCLUSION}

In dynamically changing environments monitoring groundwater levels is a critical issue. Early prediction of drought or loss of pumpage will depend on the faster response of the groundwater researchers. Timely data can be made available using advancements in wireless networking infrastructure. To build a complete, correct and practical solution to the real-time groundwater monitoring all the components of the system have to be developed and integrated efficiently. The solution outlined in this paper directly addresses one of the immediate needs of the groundwater research and provides a test bed for future research of environmental monitoring in general.

\section{REFERENCES}

[1] C.J. Taylor and W. M. Alley, "Ground-Water-Level Monitoring and the importance of Long-Term Water-Level Data," U.S. Geological Survey Circular 1217, 2001.

[2] W.B. Solley and R. R. Pierce, "Estimated use of water in the United States in 1995," U.S. Geological Survey Circular 1200, p. 71, 1998.

[3] H.R. Byun and D. A. Wilhite, "Objective Quantification of Drought Severity and Duration," Journal of Climate 12(2), pp. 742-2-756, 1999.

[4] D.A. Wilhite, "Combating Drought through Preparedness," Natural Resources Forum 26(4), pp. 275-285, 2002.

[5] D.A. Wilhite., N. J. Rosenburg and M. H. Glantz, "Improving Federal Response to Drought," Journal of Climate and Applied Meteorology 25(3), pp. 332-342, 1986.

[6] D.A. Wilhite, "State Actions to Mitigate Drought: Lessons Learned," Journal of the American Water Resources Association 33(5), pp 961-968, 1997.

[7] T.B. McKee, N. J. Doeskin and J. Kleist, "The Relationship of Drought Frequency and Duration to Time Scales," Eighth Conference on Applied Climatology, American Meteorological Society, Boston, 1993.

[8] T.B. McKee, N. J. Doeskin and J. Kleist, "Drought Monitoring with Multiple Time Scales," Ninth Conference on Applied Climatology, American Meteorological Society, Boston, MA, 1995.

[9] W.C. Palmer, "Meteorological drought," Research Paper No. 45, US Weather Bureau, Washington, DC. 1965

[10] J.A. Butterworth, R. E. Schulze, L. P. Simmonds, P. Moriarty, and F. Mugabe, "Long-Term Groundwater Level Fluctuations Due to Variation in Rainfall," Hydrology and Earth System Sciences 3(3), 1999.

[11] M.J. Neilson and D. N. Bearce, "Seasonal Variations in Water Table Elevations in the Surficial Aquifer, Birmingham Valley," Journal of the Alabama Academy of Science 69(3), pp. 175-182, 1998.

[12] W.M. Wendland, "Temporal Responses of Surface-Water and Ground-Water to Precipitation in Illinois," Journal of the American Water Resources Association 37(3), pp. 685-693, 2001.
[13] Tearfund, Water, http://www.tearfund.org/uploads/ documents/Water (1) .pdf, 2002.

[14] http://www.pldworld.com/_actel/html/ref/ glossary-security-body.htm, accessed on 3rd June 2004.

[15] http://en.wikipedia.org/wiki/802.11b, accessed on 3rd June 2004.

[16] W.W. Brent, G. F. Paul, Thomas Sisk, W. K. George, "Wireless Sensor Networks For Dense Spatio-Temporal Monitoring Of The Environment: A Case For Integrated Circuit, System, And Network Design," Proc. 2001 IEEE CAS Workshop on Wireless Communications and Networking, August 2001.

[17] B. Warneke, M. L. and B. Liebowitz, and K. Pister, "Smart dust: Communicating with a cubic-millimeter computer" IEEE Computer Magazine, pages 44-51, January 2001.

[18] http: //www. solinst. com, accessed on 3rd June 2004.

[19] http://www. unidata.com.au/product/6508.shtml, accessed on 18th June 2004.

[20] http://www.microchip.com/stellent/idcplg? IdcService $=$ SS_GET_PAGE $\&$ node $I d=1335 \&$ DocName $=$ en 010114 , accessed on 18th June 2004.

[21] http://www.altera.com/products/ip/ processors/nios/nio-index.html, accessed on 18th June 2004.

[22] http://www.dlink.com/products/?pid=241, accessed on 18th June 2004. 